\title{
Kompetenzen für die Zukunft? Eine Kritik am Lehrplan 21
}

\section{Walter Herzog}

Der gemeinsame Lehrplan der 21 deutsch-und mehrsprachigen Kantone der Schweiz ist seinem Anspruch nach an Kompetenzen orientiert. Was darunter zu verstehen ist, wird jedoch weitgehend offengelassen. Ziel des Beitrags ist die Klärung des Kompetenzverständnisses des Lehrplans 21 durch eine immanente begriffliche Kritik unter Hinzuziehung verschiedener den Lehrplan begleitender Dokumente. Es zeigt sich, dass der Kompetenzbegriff nicht nur uneinheitlich und widersprüchlich, sondern auch missverständlich und zum Teil unzutreffend verwendet wird, ein Fokus jedoch auf dem Verständnis von Kompetenz als Verbindung von Wissen und Können unter dem Anspruch der Anwendbarkeit des erworbenen Wissens liegt.

Für das schweizerische Bildungssystem erstmalig richten die Kantone, in denen Deutsch auf dem ganzen Kantonsgebiet oder in einem Teilgebiet Unterrichtssprache ist, die Ziele der obligatorischen Schule an einem gemeinsamen Lehrplan - dem Lehrplan 21 - aus. Während über dessen Umsetzung, die im föderalistischen System der Schweiz auf kantonaler Ebene erfolgt, sämtliche betroffenen Kantone bereits entschieden haben, ist die Umsetzung selber in den meisten Kantonen erst angelaufen. Als Ziel der sprachregionalen Vereinheitlichung der Lehrpläne wird die Harmonisierung des Bildungsauftrags der obligatorischen Schule genannt (D-EDK, 2013, S. 4f.). Insofern handelt es sich beim Lehrplan 21 nach offizieller Angabe nicht um eine Schulreform, sondern lediglich um die Umsetzung eines Verfassungsauftrags (D-EDK, 2010, S. 7). ${ }^{1}$

Dabei fällt allerdings auf, dass der Lehrplan 21 nicht wie bisherige Lehrpläne vorgibt, was Lehrerinnen und Lehrer zu unterrichten haben, sondern was die Schülerinnen und Schüler im Verlaufe ihrer Pflichtschulzeit zu lernen haben. Dafür steht der Begriff der Kompetenzorientierung. Durch die Formulierung von Kompetenzen würde «transparent, verständlich und nachvollziehbar» (D-EDK, 2013, S. 12) dargestellt, «welches Wissen und welche Fähigkeiten und Fertigkeiten in welcher Qualität Schülerinnen und Schüler [bis zum Ende ihrer Schulpflicht] erwerben sollen» (D-EDK, 2016, S. 25). Allein schon diese Akzentverschiebung vom Input- zum Outputbereich der Schule lässt zweifeln, dass die 
mehrfach abgegebene Versicherung, mit der "Orientierung an Kompetenzen» sei «kein Paradigmenwechsel verbunden»(D-EDK, 2013, S. 12), tatsächlich zutrifft (Künzli, 2011, Oktober, 2013, November). Die Zweifel mehren sich angesichts der uneindeutigen Verwendung des Kompetenzbegriffs. Weder im Lehrplan selber noch in seinen Begleitdokumenten findet sich eine einheitliche Definition. Zwar wird öfters umschrieben, was unter Kompetenz, Kompetenzstufen und Kompetenzaufbau zu verstehen ist, jedoch bleiben die Erläuterungen zumeist vage oder widersprechen Aussagen, die sich an anderer Stelle finden. Für einen Lehrplan, dessen politische Ambition in der Harmonisierung des Auftrags und der Ziele der obligatorischen Schule in der deutschsprachigen Schweiz liegt, stellt dies ein gravierendes Problem dar, denn die erstrebte Vereinheitlichung dürfte kaum gelingen, wenn über den Kernbegriff der Reform keine Klarheit herrscht.

Ziel der folgenden Analyse ist, die Verwendung des Kompetenzbegriffs im Lehrplan $21 \mathrm{zu}$ rekonstruieren. Methodisch ist die Analyse als immanente Kritik angelegt, d.h. der Lehrplan 21 wird nicht an einem externen Massstab gemessen, wie er sich beispielsweise in den Kompetenzbegriffen von White (1959), Roth (1971), Chomsky (1973), McClelland (1973), Loch (1979), Habermas (1984) oder Gardner (1994) findet (Herzog, 2014), sondern an seinen eigenen Ansprüchen. Dabei werden auch Texte beigezogen, die im Vor- und Umfeld des Lehrplans 21 entstanden sind.

Im ersten Schritt wird die weitverbreitete Auffassung, der Lehrplan 21 beruhe auf dem Kompetenzbegriff von Franz E. Weinert (2001a, 2001b), überprüft (1). Daran anschliessend werden schrittweise weitere Begriffsverwendungen aufgedeckt (2 bis 4). Der Beitrag schliesst mit einem knappen Ausblick (5).

\section{Der Kompetenzbegriff von Franz E. Weinert}

Gegen die Behauptung, der Lehrplan 21 würde den Kompetenzbegriff nicht klar definieren, liesse sich eine Stelle im Lehrplan anführen, an der es heisst: «Die Orientierung an Kompetenzen im Lehrplan 21 basiert u.a. auf den Ausführungen von Franz E. Weinert. Nach ihm umfassen Kompetenzen mehrere inhalts- und prozessbezogene Facetten» (D-EDK, 2016, S. 25)2, nämlich: «Fähigkeiten, Fertigkeiten und Wissen, aber auch Bereitschaften, Haltungen und Einstellungen» (ebd.). Mit Letzteren seien «neben kulturbezogenen Tugenden ... vor allem Aspekte des selbstverantwortlichen Lernens, der Kooperation, der Motivation und der Leistungsbereitschaft angesprochen»(ebd.). In dieselbe Richtung weist das Glossar, das sich im Grundlagenbericht zum Lehrplan 21 findet, wo man unter dem Lemma «Kompetenz» lesen kann: «Nach dem Pädagogen Franz E. Weinert umfassen Kompetenzen Fähigkeiten, Fertigkeiten und Wissen, aber auch Bereitschaften, Haltungen und Einstellungen, über die Schülerinnen und Schüler verfügen müssen, um neuen Anforderungssituationen 
gewachsen zu sein» (D-EDK, 2010, S. 38). Es folgt der apodiktisch formulierte Satz: «Der Lehrplan 21 stützt sich auf diesen Kompetenzbegriff» (ebd.). ${ }^{3}$

Dies kann aus verschiedenen Gründen nicht zutreffen. Erstens handelt es sich bei Weinerts (2001a, 2001b) Definition um einen rein pragmatisch begründeten Vorschlag zur Begriffsverwendung, der ohne jeden theoretischen Anspruch daherkommt. ${ }^{4}$ Als Grundlage für die Ausarbeitung eines Lehrplans kann eine theoriefreie, lediglich pragmatisch abgesicherte Definition des Kompetenzbegriffs nicht genügen.

Zweitens ist Weinerts Definition auch praktisch kaum handhabbar, da sie zu viele Aspekte umfasst. Es kann kaum erwartet werden, dass sich Kompetenzen jemals in diesem umfassenden Sinne vermitteln liessen. Indem Weinert über den kognitiven Bereich hinaus auch motivationale, volitionale, soziale und moralische Bereitschaften in den Kompetenzbegriff aufnimmt (Weinert, 2001b, S. 28), wird zudem die Grenze zwischen Unterricht und Erziehung aufgehoben und die Schule mit pädagogischen Aufgaben betraut, die ihr nicht zukommen (Herzog, 2016). Aufgrund der Konsultation zur ersten Fassung des Lehrplans 21 erteilte die Deutschschweizer Erziehungsdirektoren-Konferenz (D-EDK) denn auch den Auftrag, die Vermittlung von spezifischen Haltungen und Einstellungen zu entfernen, da sie nicht Gegenstand des Lehrplans sei (D-EDK, 2014a, S. 5f.).

Drittens kann Weinerts Definition auch insofern nicht überzeugen, weil sie eine Unterscheidung ignoriert, die in theoretischer Hinsicht unumgänglich ist, nämlich die Unterscheidung zwischen Kompetenz und Performanz. Sowohl mit Chomsky (1973) als auch mit Habermas (1983) liesse sich zeigen, dass die Rekonstruktion der Voraussetzungen kompetenten sprachlichen bzw. kommunikativen Handelns nicht hinreicht, um tatsächliches Handeln zu erklären. Die Kompetenztheorie muss um eine Performanztheorie ergänzt werden, in die jene motivationalen, volitionalen, emotionalen, sozialen und motorischen Aspekte aufgenommen werden, die bei Weinert als Teil des Kompetenzbegriffs erscheinen. Ein Kompetenzbegriff, der die Unterscheidung von Kompetenz und Performanz unterläuft, ist kaum in der Lage, einem Lehrplan eine tragfähige Basis zu geben. 5

Das lässt sich auch am Schicksal nachweisen, den der weinertsche Kompetenzbegriff bei seiner Operationalisierung zum Zweck der psychometrischen Erfassung erfährt. In ihrer erklärten Funktion, die am Output ansetzende Steuerung des Bildungssystems zu optimieren, müssen Kompetenzen messbar sein (Klieme \& Leutner, 2006, S. 877). Messen lassen sich aber vor allem die kognitiven Aspekte von Kompetenzen, kaum oder nur mit grossem Aufwand die nicht-kognitiven. Das zeigt die Klieme-Expertise, die zwar ebenfalls vorgibt, sich auf die Definition von Weinert zu stützen, aber ohne weiteres eingesteht, dass Kompetenzen «nur leistungsbezogen erfasst und gemessen werden [können]» (Klieme et al., 2003, S. 73). Auch im Schwerpunktprogramm der Deutschen Forschungsgemeinschaft zur Entwicklung von Kompetenzmodellen werden 
Kompetenzen als «kontextspezifische kognitive Leistungsdispositionen» (Klieme \& Leutner, 2006, S. 879, im Original ganze Stelle hervorgehoben) definiert. Explizit ausgeschlossen werden nicht nur die motivationalen, volitionalen, sozialen und moralischen Aspekte des Kompetenzbegriffs, wie sie in Weinerts Definition genannt werden, sondern auch überfachliche Kompetenzen.

Da der Lehrplan 21 für sich in Anspruch nimmt, leistungsorientiert zu sein (D-EDK, 2015, S. 10), ist er von den Messproblemen des Kompetenzbegriffs unmittelbar betroffen. Auch wenn eingeräumt wird, dass nicht alle Kompetenzen und Kompetenzstufen beurteilt werden müssten, ist die Entwicklung des Lehrplans 21 in direkter Orientierung an den nationalen Bildungsstandards des HarmoS-Projekts erfolgt, die im Rahmen des schweizerischen Bildungsmonitorings mittels Leistungstests überprüft werden (D-EDK, 2014b, S. 5, 20; EDK, 2004). Für die nationalen Bildungsstandards gilt explizit, dass es sich um "performance standards (handelt), also um ergebnisorientierte Leistungsstandards" (Maradan \& Mangold, 2005, S. 4). Damit wird definitiv klar, dass es nicht der Kompetenzbegriff von Weinert sein kann, auf den sich der Lehrplan 21 stützt.

\section{Ein Können, dem ein Wissen zugrunde liegt}

Wenn die Selbstauskunft, wonach der Kompetenzbegriff von Weinert dem Lehrplan 21 zugrunde liegt, nicht zutreffen kann, wie lässt sich dann ausmachen, was der Lehrplan 21 unter Kompetenz versteht? Ich werde zwei Wege gehen. Der erste Weg besteht darin, nach weiteren Erläuterungen zum Kompetenzbegriff innerhalb und ausserhalb des Lehrplans 21 zu suchen. Der zweite Weg stützt sich auf die Kompetenzen und Kompetenzstufen, wie sie in den sechs Fachbereichslehrplänen (zu «Sprachen`, «Mathematik», «Natur, Mensch, Gesellschaft», (Gestalten`, (Musik〉 sowie (Sport und Bewegung)) und den zwei Modullehrplänen (zu `Medien und Informatik` sowie ‘Berufliche Orientierung)) beschrieben werden. Wenn überhaupt, dann müsste den Bereichs- und Modullehrplänen entnommen werden können, was der Lehrplan 21 unter Kompetenz versteht. Ich werde beide Wege gehen und sie gelegentlich auch verbinden. Es wird aber immer klar sein, wann ich Erläuterungen zum Kompetenzbegriff beiziehe und wann ich Kompetenzbeschreibungen aus dem Lehrplan zitiere.

\section{Addition von Wissen und Können}

Im Lehrplan selber sowie in den Dokumenten, die ihn begleiten, finden sich verschiedene Stellen, an denen der Kompetenzbegriff umschrieben wird. In den «Rahmeninformationen zur Konsultation» des Lehrplans 21 heisst es zum Beispiel, der Lehrplan 21 beschreibe den Bildungsauftrag an die Schulen «kompetenzorientiert» (D-EDK, 2013, S. 9); es würde beschrieben, "was alle Schülerinnen und Schüler wissen und können müssen» (ebd.). Wenn dies additiv 
gemeint sein sollte, d.h. im Sinne einer Aufzählung von Wissen und Können, dann kann dies kaum als Erläuterung des Kompetenzbegriffs genommen werden. Denn Lehrpläne haben schon immer beschrieben, was Schülerinnen und Schüler nach erfolgtem Schulbesuch "wissen und können» sollen. ${ }^{6}$

Die Kombination von Wissen und Können kommt im Lehrplan 21 allerdings häufig vor. Oft gibt es auch Erweiterungen, wie die folgenden Beispiele zeigen: «Können, Wissen und Einstellungen» (D-EDK, 2016, S. 455), «Handeln, Wissen und Können» (ebd., S. 425). Und es gibt andere Begriffskombinationen, wie beispielsweise: "Wissen und Verstehen» (ebd., S. 26), "Verständnis, Wissensnutzung und Können» (ebd., S. 24), «Wissen, Fähigkeiten und Fertigkeiten» (ebd., S. 26), "Vorstellungen, Begriffe und Fähigkeiten» (ebd.), «Kenntnisse, Fertigkeiten und Routinen» (ebd., S. 208), «Fertigkeiten, Wissen und Haltungen» (ebd., S. 384), «Bereitschaften, Haltungen und Einstellungen» (ebd., S. 26). Auch wenn es an diesen Stellen oft nicht um die Erläuterung des Kompetenzbegriffs geht, sondern darum, was die Schülerinnen und Schüler lernen sollen, wird durchaus eine Aussage darüber gemacht, was unter Kompetenz zu verstehen ist. Es erstaunt daher nicht, dass wiederholt zu lesen ist, Kompetenzen hätten «mehrere» bzw. «verschiedene Facetten» (ebd., S. 11, 25, 27). Je mehr Facetten genannt werden, desto näher liegen wir bei der Definition von Weinert. Je weniger genannt werden, vor allem wenn nur Wissen und Können aufgeführt werden, desto näher liegen wir bei dem, was Lehrpläne schon immer beinhaltet haben. Weder im einen noch im anderen Fall gewinnen wir Klarheit darüber, was im Lehrplan 21 unter Kompetenz verstanden wird. Denn Kompetenz im Sinne der weinertschen Maximalvariante ist zu viel, Kompetenz im Sinne der Minimalvariante einer blossen Addition von Wissen und Können zu wenig, um einem kompetenzorientierten Lehrplan eine tragfähige begriffliche Basis zu geben.

\section{Anwendung des Gelernten}

In den "Grundlagen für den Lehrplan 21» heisst es, mit der Orientierung an Kompetenzen würde der Blick «verstärkt auf die Anwendbarkeit von Kenntnissen, Fähigkeiten und Fertigkeiten gerichtet»(D-EDK, 2010, S. 14). Es scheint, als läge hier - in der Anwendung des Gelernten - der Kerngehalt des Kompetenzbegriffs des Lehrplans, wobei wir allerdings zu beachten haben, dass von «Anwendbarkeit» und nicht von "Anwendung» die Rede ist.

Genau davon, von der Anwendung des im Unterricht Gelernten, kann man aber ebenfalls häufig lesen. Im Grundlagenkapitel des Lehrplans 21 ist von der "Anwendung deserworbenen Wissensin neuen Zusammenhängen» (D-EDK2016, S. 26) die Rede. Anderswo heisst es, dem Lehrplan 21 lasse sich entnehmen, wie Schülerinnen und Schüler «überprüfbares Wissen und Fähigkeiten erwerben, die sie in unterschiedlichen Situationen anwenden und umsetzen können» (D-EDK, 2015, S. 10). Weitere Beispiele geben die Einleitungen in die Fachbereichslehrpläne, wie zum Beispiel: «Mathematische Kompetenz zeigt sich, wenn mathe- 
matisches Wissen in konkreten Situationen angewendet wird» (D-EDK, 2016, S. 212). Nicht selten geht der Begriff der Anwendung sogar in die Beschreibung von Kompetenzen und Kompetenzstufen ein, wie zum Beispiel: "Die SuS7 können Grammatikbegriffe für die Analyse von Sprachstrukturen anwenden» (ebd., S. 96). Oder: «Die SuS können die Erkenntnisse über Energie in Alltagssituationen anwenden» (ebd., S. 321). ${ }^{8}$

Abgesehen davon, dass man dies üblicherweise Transfer nennt - im Sinne der Übertragung des in einer Situation Gelernten in eine andere oder neue Situation (Woolfolk, 2014, S. 345ff.) ${ }^{9}$-, ist unklar, ob mit dem Begriff der Anwendung mehr gemeint ist als mit dem Begriff des Könnens. So heisst es in den «Rahmeninformationen» zum Lehrplan 21, mit der Formulierung «Die Schülerinnen und Schüler können ...» werde signalisiert, dass der Lehrplan erst dann erfüllt sei, "wenn die Kinder und Jugendlichen über das nötige Wissen verfügen und dieses auch anwenden können» (D-EDK, 2014b, S. 13, Hervorh. W.H.). Massstab ist demnach nicht, dass das Wissen in neuen Situationen angewendet wird, sondern dass es überhaupt angewendet wird. Das Verb «können» soll zum Ausdruck bringen, dass das erworbene Wissen nicht «träge» bleibt (Renkl, 1996), sondern auch genutzt wird. Das Verhältnis von Wissen und Können ist daher nicht additiv, sondern multiplikativ zu verstehen: "Wissen und Können ... werden miteinander verknüpft» (D-EDK, 2016, S. 24). So scheinen es auch Klieme und Hartig (2008) zu sehen, wenn sie schreiben, Kompetenz könne verstanden werden «als die Verbindung von Wissen und Können in der Bewältigung von Handlungsanforderungen» (S. 19). Noch einen Schritt weiter geht Lersch (2007), wenn er meint: «Im Kompetenzbegriff fallen Wissen und Können zusammen» (S. 434, Hervorh. aufgehoben).

\section{Priorität des Könnens}

Dass sich Wissen und Können - und je nachdem noch weitere, psychische Kapazitäten wie Fertigkeiten oder Bereitschaften - miteinander «verknüpfen» oder «verbinden» lassen, sodass sie schliesslich «zusammenfallen», ist jedoch alles andere als trivial. Genausowenig wie Praxis aus der schlichten Anwendung von Theorie hervorgeht, entsteht Können aus der blossen Umformung von Wissen. Gemäss Neuweg (1999) gilt, dass wir einerseits vieles können, ohne dass wir auf ein (propositionales) Wissen zurückgreifen müssen, und andererseits vieles wissen, ohne dass uns in den Sinn käme, daraus ein Können zu machen. Wenn wir Ryle (1969) folgen, dann stellen Wissen (knowing that) und Können (knowing how) sogar kategorial verschiedene Begriffe dar, die sich weder über den Begriff der Anwendung noch anderswie miteinander verknüpfen lassen. Der Kompetenzbegriff scheint etwas zu versprechen, was sich gar nicht einlösen lässt.

Ausser man folgt einer anderen Spur, wonach das Können dem Wissen übergeordnet ist. Auch dafür finden sich Hinweise im Lehrplan 21, ja sie sind sogar besonders häufig anzutreffen. Denn eingeführt werden die Kompetenzen und Kompetenzstufen in den Fachbereichs- und Modullehrplänen mit der 
Standardformulierung: «Die Schülerinnen und Schüler können ...». Erst danach wird gesagt, was sie können. Kompetenzen scheinen in erster Linie ein Können zu sein, dem in zweiter Linie ein Wissen zugrunde liegt.

Das erklärt, weshalb der Kompetenzbegriff einen starken formalen Einschlag aufweist. Was die Schülerinnen und Schüler können sollen, wird mit einer Fülle an Verben umschrieben, die in der Regel auf eine Tätigkeit hinweisen. Im Vordergrund steht die Tätigkeit und nicht das Wissen, auf dem die Tätigkeit beruht. Nehmen wir ein Beispiel: «Die SuS können in eine Geschichte oder ein Thema eintauchen» (D-EDK, 2016, S. 89, Hervorh. W.H.). Abgesehen davon, dass hier eine Metapher gebraucht wird, was der angeblich transparenten, verständlichen und nachvollziehbaren Sprache, in der die Kompetenzen beschrieben sein sollen (D-EDK, 2013, S. 12), eklatant widerspricht, liegt der Fokus der Kompetenzbeschreibung auf dem Verb «eintauchen». Anhand welcher Inhalte sich das Eintauchenkönnen der Schülerinnen und Schüler manifestiert, spielt keine Rolle; sie sind gegenüber der fokussierten Tätigkeit sekundär.

Der Formalismus des Kompetenzbegriffs zeigt sich in einer Fülle an Verben, mit denen die Kompetenzen umschrieben werden. Eine Systematik ist dabei nicht ersichtlich, im Gegenteil. Oft ist nicht nachvollziehbar, weshalb ein bestimmtes Verb benutzt wird. So heisst es relativ häufig, die Schülerinnen und Schüler könnten über etwas sprechen, über etwas diskutieren, sich mit etwas auseinandersetzen, über etwas nachdenken, etwas einbeziehen, etwas vermuten, etwas kritisch reflektieren etc. Auch dafür sind Inhalte nötig, aber um welche Inhalte es sich handelt, spielt wiederum keine primäre Rolle. Gegenüber den rein formalen Tätigkeiten des Sprechens, Diskutierens, Nachdenkens oder Reflektierens ist es nicht einmal von Bedeutung, ob die Inhalte wahr oder falsch sind. ${ }^{10}$

Den Inhalten kommt die Funktion von Katalysatoren zu, die für die Entwicklung einer Kompetenz zwar notwendig sind, ihr aber äusserlich bleiben. Dem entspricht, wenn die «Beschreibung von Lernzielen in Form von Kompetenzen» (D-EDK, 2016, S. 24) damit erläutert wird, dass dadurch «Kulturinhalte mit daran zu erwerbenden fachlichen und überfachlichen Fähigkeiten und Fertigkeiten verbunden (werden)» (ebd., Hervorh. W.H.). Kompetenzorientiert unterrichten heisst, «spezifische Inhalte und Gegenstände so auszuwählen und als Lerngelegenheiten zu gestalten, dass erwünschte Kompetenzen daran erworben oder gefestigt werden können» (ebd., S. 26, Hervorh. W.H.). Das Wissen spielt insofern eine Rolle, als an ihm ein Können ausgebildet werden kann.

Damit scheinen wir dem Kompetenzbegriff des Lehrplans 21 zumindest nahegekommen zu sein. Kompetenz steht für ein Können, in das ein Wissen eingelassen ist, das für den Aufbau des Könnens wesentlich, aber relativ beliebig ist, denn begrifflich ausschlaggebend für eine Kompetenz ist nicht, dass sie erworben wird, sondern genutzt werden kann.

Noch zu präzisieren wäre allerdings, was für Situationen gemeint sind, in denen von den schulisch erworbenen Kompetenzen Gebrauch gemacht wird. 
Sind es wiederum schulische Situationen (im selben oder in einem anderen Fach)? Oder sind es ausserschulische Situationen? Die Fragen lassen sich nicht eindeutig beantworten. Ausdrücke wie Anwendung, Umsetzung oder Gebrauch (des Gelernten) in "verschiedenen» (D-EDK, 2016, S. 478), "unterschiedlichen» (ebd., S. 60), «authentischen» (ebd., S. 63f.), «konkreten» (ebd., S. 212), «neuen» (ebd., S. 81) oder "ungewohnten» (ebd., S. 32) Situationen, wie sie häufig vorkommen, sind in Bezug auf die Frage, ob «für die Schule» oder «für das Leben" gelernt wird, meist unbestimmt. Es hilft auch nicht, sich bei Tenorth (2004) zu erkundigen, der im Kompetenzbegriff die Tatsache verkörpert sieht, «dass die Lernenden Fähigkeiten in einer Weise erworben haben, die es erlauben, sie von der Lern- und Ursprungssituation abzulösen und in der Welt selbst anzuwenden» (S. 110). Denn im Lehrplan 21 finden sich nur wenige Stellen, die eindeutig bestätigen würden, dass mit der Anwendung des schulisch Gelernten (auch) die ausserschulische Welt gemeint ist. ${ }^{11}$

\section{Kompetenzen, die keine sind}

Selbst wenn dies der Bedeutungskern des Kompetenzbegriffs ist, wie er im Lehrplan 21 verwendet wird, sind wir mit unserer Analyse noch nicht am Ende. Denn nicht nur die schiere Vielfalt an Verben, mit denen die von den Schülerinnen und Schülern zu erwerbenden Kompetenzen umschrieben wird, auch die Tatsache, dass die Kompetenzen keineswegs immer in Könnenssätzen daherkommen, wecken Zweifel an der Schlüssigkeit unserer Rekonstruktion. Zudem gibt es sprachlich eigenwillige Formulierungen, welche die Frage aufwerfen, ob der Lehrplan 21 wirklich durchgängig kompetenzorientiert ist.

\section{Ist auch das Wissen ein Können?}

Wie muss man den folgenden Satz verstehen: «Wissen als Kompetenz wird in einem breiten Sinne verstanden: als direkt nutzbares Verfügungswissen, als Reflexionswissen und als Orientierungswissen» (D-EDK, 2016, S. 24)? Ist er so zu verstehen, dass es Wissensformen gibt, die nicht in ein Können eingebunden, sondern selber schon Kompetenzen sind?

Tatsächlich gibt es im Lehrplan 21 Kompetenzbeschreibungen, die ausschliesslich auf Wissen ausgerichtet sind. So zum Beispiel in den folgenden Fällen: «Die SuS wissen, dass Kenntnisse, die sie über die französischsprachigen / englischsprachigen / italienischsprachigen Kulturen haben, häufig klischeehafte Aspekte beinhalten» (ebd., S. 128, 156, 180). «Die SuS wissen, wie Energie unter verschiedenen Rahmenbedingungen gespeichert und transportiert werden kann» (ebd., S. 321). Indirekte Hinweise auf bblosses, Wissen finden sich im Fachbereichslehrplan «Mathematik», wie zum Beispiel: «Die SuS verstehen und verwenden arithmetische Begriffe und Symbole» (ebd., S. 216). Um was anderes als um Wissen handelt es sich, wenn man einen Begriff oder ein Symbol versteht und (korrekt) 
verwendet? Wie es zutreffend heisst, bilden Begriffe die «Bausteine des Wissens» (ebd., S. 253).

Eine spezifische Form des Gebrauchs von Begriffen ist das Benennen, wofür sich im Lehrplan 21 viele Belege finden, wie zum Beispiel: «Die SuS können Kreis, Dreieck, Rechteck, Quadrat, Würfel und Kugel benennen» (ebd., S. 226). Oder: «Die SuS können Unterschiede im Körperbau von Mädchen und Knaben mit angemessenen Wörtern benennen» (ebd., S. 274). Auch wer etwas (korrekt) benennen kann, zeigt nicht in erster Linie, dass er etwas kann, sondern dass er etwas weiss. 12

Weitere Beispiele für verkappte Wissensformulierungen gibt das ebenfalls häufig verwendete Verb «kennen». Hier geht es nicht darum, dass die Schülerinnen und Schüler etwas können, sondern dass sie mit etwas vertraut sind. Zum Beispiel: «Die SuS kennen die Bibliothek als Ort mit reichhaltigem Leseangebot» (ebd., S. 78), «Die SuS kennen den Begriff Partikel» (ebd., S. 96) oder «Die SuS kennen die Produkte des kleinen Einmaleins» (ebd., S. 218). Semantisch gehört Kennen zum Wortfeld Wissen, weshalb auch hier gilt, dass der Lehrplan 21 keineswegs tel quel an Kompetenzen orientiert ist, sondern eine nicht geringe Anzahl von Lernzielen enthält, die sblosses) Wissen beinhalten. ${ }^{13}$

\section{Unsinnige Lernziele}

Der Lehrplan 21 enthält jedoch nicht nur Könnensformulierungen, die sich als kaschierte Wissensansprüche entpuppen, sondern auch Lernziele, die in einem Lehrplan sprachlich keinen Sinn machen. Das gilt vor allem für Kompetenzund Kompetenzstufenbeschreibungen, in denen Verben wie "wahrnehmen», «erleben» und «erfahren» vorkommen.

Sehen wir uns einige Beispiele an: «Die SuS können Gehörtes wahrnehmen» (D-EDK, 2016, S. 433), «Die SuS können eigene Emotionen wahrnehmen» (ebd., S. 474) oder «Die SuS können ... Zeitdauer erleben» (ebd., S. 302). Können Wahrnehmen und Erleben Fähigkeiten im Sinne von Dispositionen sein? Zu sagen, man könne (etwas) wahrnehmen, meint, dass keine äusseren Umstände gegeben sind, die einen daran hindern, (etwas) zu sehen, zu hören oder anderswie wahrzunehmen. Auch eine physische Beeinträchtigung (wie Schwerhörigkeit oder Sehschwäche) kann Anlass für eine entsprechende Äusserung sein. Subjektiv können wir jedoch nicht versuchen, eine Wahrnehmung oder ein Erlebnis zu haben, da es sich dabei nicht um Leistungen, sondern um Vorgänge bzw. Widerfahrnisse handelt (Straus, 1978).

Ähnlich liegt der Fall bei Erfahrungen. Auch hier zunächst einige Beispiele: "Die SuS können Erfahrungen sammeln mit ...» (D-EDK, 2016, S. 87, 95, 100f.) oder «Die SuS können technische Zusammenhänge spielerisch erfahren» (ebd., S. 406). Was ist der Unterschied zwischen «Erfahrungen sammeln» und "Erfahrungen sammeln können»? Das Können kann sich hier nicht darauf beziehen, dass den Schülerinnen und Schülern ermöglicht wird, Erfahrungen zu machen. Denn dies wäre eine didaktische Anweisung und nicht eine Lernziel- 
beschreibung. Ähnlich wie Wahrnehmungen und Erlebnisse sind Erfahrungen nicht Leistungen, zu denen man aufgefordert werden und die man willentlich erbringen kann, sondern Ereignisse, von denen man betroffen wird (Gadamer, 1975, S. 334ff.). In einem Lehrplan, der erklärtermassen leistungsorientiert ist (D-EDK, 2015, S. 10), machen solche Kompetenzbeschreibungen daher keinen Sinn. Zu verdanken sind sie vermutlich dem eng geschnürten Korsett der Könnensformulierungen. ${ }^{14}$

\section{Kompetenz: substantivisch oder adjektivisch?}

Wenn wir uns nochmals der Definition von Weinert zuwenden, dann fällt auf, dass der Kompetenzbegriff in einer eigenartigen Doppeldeutigkeit verwendet wird. Unter Kompetenzen versteht Weinert (2001b) nämlich nicht nur psychische Dispositionen und deren Gebrauch bei der Lösung von Problemen, sondern auch die «Bereitschaften und Fähigkeiten, um die Problemlösungen in variablen Situationen erfolgreich ... nutzen zu können» (S. 27f., Hervorh. W.H.). Kompetenz ist einerseits ein Substantiv, das zur Bezeichnung einer Disposition verwendet wird, und andererseits ein Adjektiv, mit dem das Substantiv qualifiziert wird, wobei die beiden Bedeutungen in ein und derselben Definition vorkommen.

Auch im Lehrplan 21 wird der Kompetenzbegriff doppelt verwendet, also nicht nur substantivisch, sondern auch adjektivisch. So heisst es zum Beispiel: «Der Bedarf nach kompetent Englisch Sprechenden wächst» (D-EDK, 2016, S. 58). Die Schülerinnen und Schüler werden unterstützt, «um sich kompetent in Kultur und Gesellschaft zu orientieren» (ebd., S. 262). Zwar sind diese Stellen nicht sehr häufig, aber es fehlt jede Begründung für die doppelte Verwendungsweise des Begriffs. Pädagogisch ist dies heikel, da es die Überlagerung von Kompetenz als Lernziel und Kompetenz als Ausmass der Zielerreichung erschwert, Schülerleistungen angemessen zu beurteilen.

Grundsätzlich stellt sich die Frage, ob der Kompetenzbegriff nicht überhaupt auf der Substantivierung eines Adjektivs beruht und es genau deshalb so schwer fällt, den Begriff klar zu definieren. Im alltäglichen Sprachgebrauch nennen wir eine Person kompetent, wenn sie Leistungen erbringt, die wir als hochstehend oder zumindest als sachlich angemessen beurteilen. Dabei meinen wir nicht, die Person verfüge über eine spezifische Kompetenz, sondern unterstellen, dass sie die Fähigkeiten und Fertigkeiten sowie das Wissen und Können hat, um die betreffende Tätigkeit zufriedenstellend auszuführen. Auch wenn ein Autor wie Dornes (2001) einem Buch über die frühkindliche Entwicklung den Titel «Der kompetente Säugling» gibt, meint er nicht, dass der Säugling spezifische Kompetenzen hat, sondern in einem Ausmass kompetent ist, das bisher unterschätzt wurde.

Dies scheint auch im Englischen die häufigere Verwendung zu sein, wie ein beliebig herausgegriffenes Zitat eines amerikanischen Psychologen zeigt, der 
zum Kompetenzbegriff meint: «To be competent in a domain signifies a degree of skills and knowledge ... higher than those of the novice, but not necessarily as high as a master" (Simonton, 2003, S. 231). ${ }^{15}$ Dem entspricht, wenn Dreyfus und Dreyfus (1988) den Kompetenzbegriff dazu verwenden, um eine Stufe des Erwerbs von Fertigkeiten zu benennen, nämlich die dritte von fünf Stufen. ${ }^{16}$ Wird der Kompetenzbegriff adjektivisch und nicht substantivisch gebraucht, können die Begriffe Kompetenz und Performanz sogar in einem Atemzug genannt werden, wie bei Glaser (1976, S. 8f.), der von einer competent performance und einem competent performer spricht.

\section{Wird die Umsetzung Klarheit bringen?}

Im Ergebnis zeigt unsere Analyse, dass der Kompetenzbegriff im Lehrplan 21 uneinheitlich und widersprüchlich verwendet wird. Die offizielle Angabe, wonach der weinertsche Kompetenzbegriff die Grundlage des Lehrplans 21 bildet, kann nicht zutreffen, Erläuterungen zum Kompetenzbegriff sind oft vage und unverbindlich, in den Bereichs- und Modullehrplänen werden häufig nicht Kompetenzen beschrieben, sondern kaschierte Wissensansprüche gestellt, und der Zwang zur Darstellung der Kompetenzen in Form von Könnenssätzen erzeugt sprachlich inkorrekte Formulierungen. Zwar lässt sich vermuten, dass der Kern des Kompetenzbegriffs im Lehrplan 21 in einer Verbindung von Wissen und Können liegt, bei der das Können dem Wissen übergeordnet ist, damit die Gewähr besteht, dass das schulisch Gelernte auch angewendet wird. Dabei bleibt aber offen, was unter Anwendung im Unterschied zu Transfer genau zu verstehen ist und in welchen Situationen die erworbenen Kompetenzen angewendet werden sollen: in schulischen oder in ausserschulischen Situationen?

Dass der Kompetenzbegriff in unterschiedlicher Bedeutung in Gebrauch ist, stellt allerdings keine neue Erkenntnis dar (Maag Merki, 2009; Straub, 2007). Schon 2001 sprach Westera in Bezug auf die pädagogische Verwendung des Begriffs von einer babylonischen Sprachverwirrung und stellte fest: "Competence is no more than an unclear label and does not increase our knowledge and understanding of the world» (S. 86). 2006 wurde die Diagnose von Crahay bekräftigt: "Le statut scientifique du concept de compétences est incertain» (S. 107). Dass der Begriff innerhalb ein und desselben Dokuments wie dem Lehrplan 21 aber so uneinheitlich verwendet wird, wirft die Frage auf, welche Leistung dem Kompetenzbegriff eigentlich zugedacht ist. Kann der Lehrplan 21 überhaupt herbeiführen, was ihm herbeizuführen aufgetragen ist, nämlich den Unterricht auf Kompetenzorientierung umzustellen, wenn sein Kernbegriff dermassen unklar ist?

Wenn Westera (2001) dem Kompetenzbegriff den Status eines Labels zumisst und Künzli (2013, November) in gleichem Sinn von einem Slogan spricht, dann fragt sich, ob dem Begriff überhaupt eine pädagogische oder nicht vielmehr eine 
politische Funktion zukommt. Der Kompetenzbegriff scheint einer Reformbewegung als Signet zu dienen, um auf die Akzentverschiebung vom Lehren zum Lernen aufmerksam zu machen und für den Wechsel von der input- zur outputorientierten Steuerung des Bildungssystems Akzeptanz zu schaffen (Herzog, 2013). Diese Mobilisierungsleistung lässt sich mit einem diffusen Begriff eher erbringen als mit einem präzisen.

$\mathrm{Ob}$ dies allerdings eine zutreffende Erklärung für die Vagheit des Kompetenzbegriffs im Lehrplan 21 ist, lässt sich aufgrund der vorausgehenden Analyse nicht entscheiden. So oder so wird es nun darum gehen, den Lehrplan 21 in die schulische Praxis umzusetzen. Dabei stehen im Vordergrund die Entwicklung von Lehrmitteln (die bereits im Gang ist), die Ausarbeitung von Vorgaben zur Schülerbeurteilung (die in der Verantwortung der Kantone liegt), weitere Handreichungen zur Umsetzung des Lehrplans (wie sie seitens der kantonalen Bildungsverwaltungen und der Pädagogischen Hochschulen teilweise bereits vorliegen), die Anpassung der Grundausbildung und Weiterbildung der Lehrpersonen an den neuen Lehrplan und die Überprüfung der Schülerleistungen mittels standardisierter Tests (wie sie im Rahmen des schweizerischen Bildungsmonitorings vorgesehen ist). Ob sich für den Kompetenzbegriff im Verlauf dieser Umsetzungsarbeiten mehr Klarheit ergeben wird, lässt sich jedoch nur schwer abschätzen. Voraussetzung dafür wäre, dass die politische Funktion des Kompetenzbegriffs hinter seine pädagogische zurücktritt. Denn nur so kann erwartet werden, dass der aktuelle Zustand als unbefriedigend empfunden wird und der Kompetenzbegriff zuhanden derjenigen, die den Lehrplan 21 in die Schulpraxis umsetzen müssen, einer Klärung zugeführt wird.

\section{Anmerkungen}

1 Gemeint ist Art. 62 der Schweizerischen Bundesverfassung, der u.a. eine «Harmonisierung ... der Ziele der Bildungsstufen» verlangt.

2 Ich zitiere den Lehrplan 21 nach der Gesamtausgabe, und zwar nach der «(b)ereinigte(n) Fassung vom 29.02.2016» (D-EDK, 2016, Innenseite des Deckblatts).

3 In einem Grundlagenpapier zum Lehrplan 21 heisst es zudem: «Der Lehrplan geht vom selben Kompetenzbegriff aus wie HarmoS» (Weber, 2007, S. 11). HarmoS stützt sich aber explizit auf die Definition von Weinert (EDK, 2004, S. 8). In den Aufträgen zur Überarbeitung des Lehrplans 21 heisst es: «Kompetenzdefinition F. Weinert beibehalten» (D-EDK, 2014a, S. 8), was so gelesen werden kann, dass auch von der Projektträgerschaft angenommen wird, der Lehrplan 21 basiere auf dem weinertschen Kompetenzbegriff.

4 Weinert (2001a) spricht von «a pragmatic definition of the concept of competence [which] has practical rather than theoretical utility» (S. 63).

5 Von Performanz als begrifflichem Gegenstück zu Kompetenz ist im Lehrplan 21 an keiner Stelle die Rede. Auch im Glossar zum Lehrplan 21 kommt der Begriff nicht vor (D-EDK, 2010, S. 37ff.). An den drei Stellen, an denen in den Fachbereichslehrplänen 'Gestalten` und «Musik» von «Performance» die Rede ist (D-EDK, 2016, S. 399, 442, 443), wird der Begriff offensichtlich in anderer Bedeutung verwendet.

6 Im Englischen ist in der Regel von «knowledge and skills» die Rede. So auch in den PISA-Studien. In Bezug auf die drei von PISA erfassten Kompetenzbereiche Lesen, Mathematik und Naturwissenschaften heisst es beispielsweise: «PISA aims to define each 
domain not merely in terms of mastery of the school curriculum, but in terms of important knowledge and skills needed in adult life» (Organisation for Economic Co-operation and Development [OECD], 1999, S. 8).

7 Um den Text nicht zu überladen, wird die Phrase, mit der sämtliche Kompetenz- und Kompetenzstufenbeschreibungen eingeleitet werden, nämlich «Die Schülerinnen und Schüler», mit «Die SuS» abgekürzt.

8 Nicht weit entfernt vom Begriff der «Anwendung» ist derjenige der «Verwendung», der in den Bereichslehrplänen ‘Sprachen` und «Mathematik» vergleichsweise häufig vorkommt. Auch Verben wie «nutzen», «ausführen» oder «umsetzen» kommt eine ähnliche Funktion $\mathrm{zu}$.

9 Interessanterweise finden sich im Bereichslehrplan «Sprachen` mehrere Stellen, an denen ausdrücklich von Transfer die Rede ist. Unter der Überschrift «Didaktische Hinweise» heisst es, die Effizienz des Sprachenlernens werde gesteigert, "wenn die Schülerinnen und Schüler befähigt werden, das Transferpotenzial zwischen den Sprachen bewusst zu nutzen» (D-EDK, 2016, S. 60). Der Transferbegriff ist sogar in einzelne Kompetenzbeschreibungen eingegangen, wie zum Beispiel: «Die SuS können ... Sprechstrategien aus Deutsch und anderen Sprachen ... transferieren und einsetzen» (ebd., S. 115, vgl. auch S. 143, 168). Analoge Beispiele finden sich zum Transfer von Hörstrategien, Schreibstrategien und Lesestrategien (ebd., S. 106, 110, 119, 134, 138, 147, 161, 164, 172).

10 Allerdings finden sich an einigen Stellen doch qualifizierende Attribute, wie zum Beispiel: «Die SuS können Zeitbegriffe aufbauen und korrekt verwenden» (D-EDK, 2016, S. 302), «Die SuS können ihre Sprechmotorik, Artikulation, Stimmführung angemessen nutzen» (ebd., S. 81) oder «Die SuS können Alltagsgeräte sachgemäss und sicher bedienen» (ebd., S. 419).

11 Zum Beispiel: «Auf diese Weise gewinnen [die Schülerinnen und Schüler] Erkenntnisse und erwerben Werkzeuge, die sie zur Lösung von Aufgaben im Unterricht und im Alltag nutzen können» (D-EDK, 2016, S. 21). Oder: «Auf diese Weise werden Handlungskompetenzen erworben, die auch in neuen Problemstellungen im Unterricht und im Alltag angewendet werden können» (ebd., S. 385).

12 Auch das Verb «nennen» kommt häufig vor und erfüllt eine ähnliche Funktion, wie die folgenden Beispiele zeigen: «Die SuS können Grundlagen für die Gesunderhaltung des Körpers nennen» (D-EDK, 2016, S. 274), «Die SuS können Rechte und Pflichten von Individuen in unserer Gesellschaft nennen» (ebd., S. 307) oder «Die SuS können Regeln nennen» (ebd., S. 474).

13 Es wäre interessant, weiteren Verben nachzugehen und zu untersuchen, wie weit es sich auch dabei um sinnverwandte Wissensformulierungen handelt. Kandidaten wären: beschreiben, darstellen, unterscheiden, darlegen, aufzeigen, aufzählen, über etwas eine Aussage machen, eine Zuordnung vornehmen, vergleichen, erklären, etwas erkennen etc.

14 Auch hier läge es nahe, analog gelagerten Fällen nachzugehen, wie zum Beispiel: «Die SuS können verstehen» (D-EDK, 2016, S. 303, 306, 322), «Die SuS können entdecken» (ebd., S. $105,137,287$ ) oder «Die SuS können üben» (ebd., S. 430, 438, 445).

15 Analog definiert die American Psychological Association (APA) competence wie folgt: "Possession of sufficient skills, knowledge, or qualities as required in a given situation" (APA, 2001, S. 54).

16 Im Einzelnen werden die Stufen umschrieben mit: Novize (novice), fortgeschrittener Anfänger (advanced beginner), Kompetenz (competent), Gewandtheit (proficient) und Experte (expert). Im Deutschen ist oft von einem Modell der Kompetenzentwicklung die Rede, im englischen Original sprechen die Gebrüder Dreyfus aber von einem «model of skill acquisition». 


\section{Literaturverzeichnis}

APA. (2001). Thesaurus of Psychological Index Terms (9th ed.). Washington, DC: American Psychological Association.

Chomsky, N. (1973). Aspekte der Syntax-Theorie. Frankfurt a.M.: Suhrkamp.

Crahay, M. (2006). Dangers, incertitudes et incomplétude de la logique de la compétence en éducation. Revue Française de Pédagogie, 154, 97-110.

D-EDK. (2010). Grundlagen für den Lehrplan 21. Verabschiedet von der Plenarversammlung der deutschsprachigen EDK-Regionen am 18. März 2010. Geschäftsstelle der deutschsprachigen EDK-Regionen. Zugriff am 19.05.2018 unter http://www.lehrplan.ch/sites/ default/files/Grundlagenbericht.pdf

D-EDK. (2013). Rahmeninformationen zur Konsultation. Luzern: Deutschschweizer Erziehungsdirektoren-Konferenz. Zugriff am 19.05.2018 unter http://www.nw.ch/dl.php/ de/51dfc4fde6314/Rahmeninformation_zur_Konsultation.pdf

D-EDK. (2014a). Aufträge zur Überarbeitung des Lehrplans 21. Luzern: Deutschschweizer Erziehungsdirektoren-Konferenz. Zugriff am 19.05.2018 unter http://www.lehrplan.ch/ sites/default/files/zusammenfassung_ueberarbeitungsauftraege_0.pdf

D-EDK. (2014b). Rahmeninformationen. Luzern: Deutschschweizer Erziehungsdirektoren-Konferenz. Zugriff am 19.05.2018 unter https://www.lehrplan.ch/sites/default/files/ lp21_rahmeninformation_\%202014-11-06.pdf

D-EDK. (2015). Schlussbericht zum Projekt Lehrplan 21. Luzern: Deutschschweizer Erziehungsdirektoren-Konferenz. Zugriff am 19.05.2018 unter https://www.lehrplan.ch/sites/ default/files/Schlussbericht\%20Projekt\%20Lehrplan\%2021_2015-06-18.pdf

D-EDK. (2016). Lehrplan 21. Gesamtausgabe (bereinigte Fassung vom 29.02.2016). Luzern: Deutschschweizer Erziehungsdirektoren-Konferenz. Zugriff am 19.05.2018 https://v-ef.lehrplan.ch/container/V_EF_DE_Gesamtausgabe.pdf

Dornes, M. (2001). Der kompetente Säugling. Die präverbale Entwicklung des Menschen. Frankfurt a.M.: Fischer.

Dreyfus, H. L. \& Dreyfus, S. E. (1988). Künstliche Intelligenz. Von den Grenzen der Denkmaschine und dem Wert der Intuition. Reinbek: Rowohlt.

EDK. (2004). HARMOS. Zielsetzungen und Konzeption. Juni 2004. Bern: Schweizerische Konferenz der kantonalen Erziehungsdirektoren. Zugriff am 19.05.2018 unter http:// www.edudoc.ch/static/web/arbeiten/harmos/weissbuch_d.pdf

Gadamer, H.-G. (1975). Wahrheit und Methode. Grundzüge einer philosophischen Hermeneutik (4. Aufl.). Tübingen: Mohr.

Gardner, H. (1994). Abschied vom IQ. Die Rahmen-Theorie der vielfachen Intelligenzen. Stuttgart: Klett-Cotta.

Glaser, R. (1976). Components of a Psychology of Instruction: Toward a Science of Design. Review of Educational Research, 46, 1-24.

Habermas, J. (1983). Moralbewusstsein und kommunikatives Handeln. Frankfurt a.M.: Suhrkamp.

Habermas, J. (1984). Vorstudien und Ergänzungen zur Theorie des kommunikativen Handelns. Frankfurt a.M.: Suhrkamp.

Herzog, W. (2013). Bildungsstandards - eine kritische Einführung. Stuttgart: Kohlhammer.

Herzog, W. (2014). Anthropologik des Könnens. Werner Loch als Pionier der Kompetenzorientierung? Pädagogische Rundschau, 68, 573-590.

Herzog, W. (2016). Der Erziehungsauftrag von Lehrerinnen und Lehrern. In M. Rothland (Hrsg.), Beruf Lehrer/Lehrerin. Ein Studienbuch (S. 171-185). Münster: Waxmann.

Klieme, E. \& Hartig, J. (2008). Kompetenzkonzepte in den Sozialwissenschaften und im erziehungswissenschaftlichen Diskurs. In M. Prenzel, I. Gogolin \& H.-H. Krüger (Hrsg.), Kompetenzdiagnostik (S. 11-29). Wiesbaden: Verlag für Sozialwissenschaften. 
Klieme, E. \& Leutner, D. (2006). Kompetenzmodelle zur Erfassung individueller Lernergebnisse und zur Bilanzierung von Bildungsprozessen. Zeitschrift für Pädagogik, 52, 876-903.

Klieme, E., Avenarius, H., Blum, W., Döbrich, P., Gruber, H., Prenzel, M., Reiss, K., Riquarts, K., Rost, J., Tenorth, H.-E. \& Vollmer, H. J. (2003). Zur Entwicklung nationaler Bildungsstandards. Eine Expertise. Bonn: Bundesministerium für Bildung und Forschung.

Künzli, R. (2011, Oktober). Lehrplan 21 - ein bildungspolitisches Projekt? Referat an der PH Zürich. Zugriff am 19.05.2018 unter http://www.lehrplanforschung.ch/wp-content/ uploads/2011/09/Vortrag-PHZH_VM21.pdf

Künzli, R. (2013, November). Kompetenzen in Lehrplänen - eine hoffnungsfrohe Systemreform. Referat vor der Aargauischen Kantonalen Lehrerinnen- und Lehrerkonferenz. Zugriff am 19.05.2018 unter http://www.lehrplanforschung.ch/wp-content/uploads/2013/07/ Kompetenzen-in-Lehrpl\%C3\%A4nen-eine-hoffnungsfrohe-Systemreform-Vortrag-Aarau.pdf

Lersch, R. (2007). Unterricht und Kompetenzerwerb. In 30 Schritten von der Theorie zur Praxis kompetenzfördernden Unterrichts. Die Deutsche Schule, 99, 434-446.

Loch, W. (1979). Curriculare Kompetenzen und pädagogische Paradigmen. Bildung und Erziehung, 32, 241-266.

Maag Merki, K. (2009). Kompetenz. In S. Andresen, R. Casale, T. Gabriel, R. Horlacher, S. Larcher Klee \& J. Oelkers (Hrsg.), Handwörterbuch Erziehungswissenschaft (S. 492-506). Weinheim: Beltz.

Maradan, O. \& Mangold, M. (2005). Bildungsstandards in der Schweiz: Das Projekt HarmoS. ph-akzente, Heft 2, 3-7.

McClelland, D. C. (1973). Testing for competence rather than for «intelligence». American Psychologist, 28, 1-14.

Neuweg, G. H. (1999). Könnerschaft und implizites Wissen. Zur lehr-lerntheoretischen Bedeutung der Erkenntnis- und Wissenstheorie Michael Polanyis. Münster: Waxmann.

OECD. (1999). Measuring student knowledge and skills: A new framework for assessment. Paris: Organisation for Economic Co-operation and Development.

Renkl, A. (1996). Träges Wissen: Wenn Erlerntes nicht genutzt wird. Psychologische Rundschau, 47, 78-92.

Roth, H. (1971). Pädagogische Anthropologie, Bd. II: Entwicklung und Erziehung. Hannover: Schroedel.

Ryle, G. (1969). Der Begriff des Geistes. Stuttgart: Reclam.

Simonton, D. K. (2003). Expertise, competence, and creative ability: The perplexing complexities. In R. J. Sternberg \& E. L. Grigorenko (Eds.), Perspectives on the Psychology of Abilities, Competencies, and Expertise (S. 213-239). New York, NY: Cambridge University Press.

Straub, J. (2007). Kompetenz. In J. Straub, A. Weidemann \& D. Weidemann (Hrsg.), Handbuch interkulturelle Kommunikation und Kompetenz (S. 35-46). Stuttgart: J. B. Metzler.

Straus, E. (1978). Vom Sinn der Sinne. Ein Beitrag zur Grundlegung der Psychologie (2. Aufl.). Berlin: Springer.

Tenorth, H.-E. (2004). Bildungsziele, Bildungsstandards und Kompetenzmodelle. In D. Diskowski \& E. Hammes-Di Bernardo (Hrsg.), Lernkulturen und Bildungsstandards. Kindergarten und Schule zwischen Vielfalt und Verbindlichkeit (S. 105-115). Baltmannsweiler: Schneider Verlag Hohengehren.

Weber, A. (2007). Hearing konzeptuelle Grundlagen Deutschschweizer Lehrplan: Hintergrundbericht (Stand: 30.10.2007). Luzern: Projektleitung Deutschschweizer Lehrplan.

Weinert, F. E. (2001a). Concept of competence: A conceptual clarification. In D. S. Rychen \& L. H. Salganik (Hrsg.), Defining and Selecting Key Competencies (S. 45-65). Seattle, WA: Hogrefe \& Huber. 
Weinert, F. E. (2001b). Vergleichende Leistungsmessung in Schulen - eine umstrittene Selbstverständlichkeit. In Ders. (Hrsg.), Leistungsmessungen in Schulen (S. 17-31). Weinheim: Beltz.

Westera, W. (2001). Competences in education: a confusion of tongues. Journal of Curriculum Studies, 33, 75-88.

White, R. W. (1959). Motivation reconsidered: The concept of competence. Psychological Review, 66, 297-333.

Woolfolk, A. (2014). Pädagogische Psychologie (12. Aufl.). Hallbergmoss: Pearson.

Schlagworte: Kompetenz, Können, Lehrplan, Schulreform, Wissen

\section{Compétences pour l'avenir? Une critique du plan d'études 21}

\section{Résumé}

Le plan d'études commun aux 21 cantons suisses germanophones et plurilingues se base sur une approche par compétences. Ce que cela signifie exactement est toutefois laissé en grande partie ouvert. L'objectif de cet article est l'élucidation de l'idée de compétences propre au plan d'études 21 à travers une critique conceptuelle intrinsèque, tout en ayant recours aux différents documents qui l'accompagnent. L'analyse réalisée montre que le terme de compétence est non seulement utilisé de façon hétéroclite et contradictoire, mais également équivoque et partiellement incorrecte. En même temps, une attention est portée à la compréhension de la compétence comme combinaison de savoirs et savoirfaire, revendiquant l'applicabilité des connaissances acquises.

Mots-clés: Compétence, savoir-faire, plan d'études, réforme scolaire, savoir

\section{Competenze per il futuro? Una critica del piano di studio 21}

\section{Riassunto}

Il piano di studio comune ai 21 cantoni svizzeri germanofoni e multilingui della Svizzera si basa su un approccio per competenze. Tuttavia, il significato di un approccio per competenze rimane in gran parte indefinito. Mediante una critica concettuale immanente basata su vari documenti che accompagnano il piano di studio 21, l'articolo verte a chiarire l'idea di competenza così come viene intesa nel piano di studio. Dall'analisi appare che la nozione di competenza non è solo usata in un modo eterogeneo e contraddittorio, ma anche in modo equivoco e parzialmente scorretto. Nel contempo, l'accento è messo sulla comprensione della competenza come combinazione di abilità e conoscenze che rivendicano l'applicabilità delle conoscenze acquisite.

Parole chiave: Competenza, abilità, curriculum, riforma scolastica, conoscenza 


\section{Competences for the Future? A Critical Review of the New Swiss German Curriculum}

\section{Summary}

The new curriculum shared by the 21 Cantons of Switzerland which are German-speaking or multilingual («Lehrplan 21») claims to be competence oriented. However, the exact signification of this claim is open to interpretation. This contribution aims to clarify the Lehrplan 21's understanding of competence by means of an immanent conceptual critique and by referring to various accompanying documents. It will be shown that the term competence is not only used in an incoherent and contradictory manner, but also incomprehensibly and partially incorrectly. Also the focus is placed on the understanding of competence as a combination of skills and knowledge assuming the applicability of the acquired knowledge.

Keywords: Competence, skills, curriculum, school reform, knowledge 
